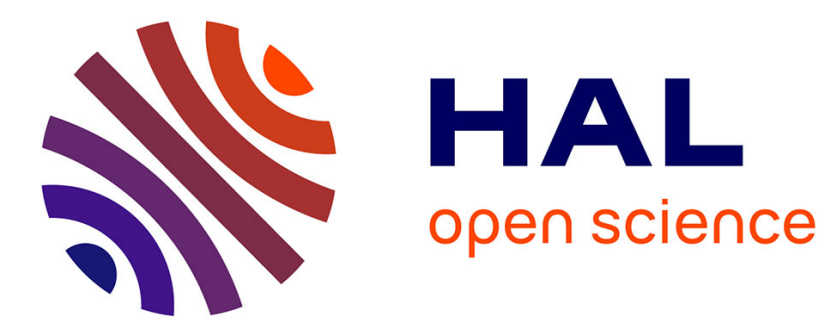

\title{
Excited-state symmetry breaking of linear quadrupolar chromophores: A transient absorption study
} Nadia Dozova, Lionel Ventelon, Guillaume Clermont, Mireille Blanchard-Desce, Pascal Plaza

\section{- To cite this version:}

Nadia Dozova, Lionel Ventelon, Guillaume Clermont, Mireille Blanchard-Desce, Pascal Plaza. Excitedstate symmetry breaking of linear quadrupolar chromophores: A transient absorption study. Chemical Physics Letters, 2016, 664, pp.56 - 62. 10.1016/j.cplett.2016.10.020 . hal-01382724

\section{HAL Id: hal-01382724 https://hal.sorbonne-universite.fr/hal-01382724}

Submitted on 17 Oct 2016

HAL is a multi-disciplinary open access archive for the deposit and dissemination of scientific research documents, whether they are published or not. The documents may come from teaching and research institutions in France or abroad, or from public or private research centers.
L'archive ouverte pluridisciplinaire HAL, est destinée au dépôt et à la diffusion de documents scientifiques de niveau recherche, publiés ou non, émanant des établissements d'enseignement et de recherche français ou étrangers, des laboratoires publics ou privés. 


\title{
Excited-state symmetry breaking of linear quadrupolar chromophores:
}

\section{A transient absorption study}

Nadia Dozova, ${ }^{\mathrm{a}, \mathrm{b}}$ Lionel Ventelon, ${ }^{\mathrm{a}, \mathrm{b}}$ Guillaume Clermont, ${ }^{\mathrm{c}}$ Mireille Blanchard-Desce, ${ }^{\mathrm{c}}$ Pascal Plaza ${ }^{\mathrm{a}, \mathrm{b}} *$

${ }^{a}$ Ecole normale supérieure, PSL Research University, UPMC Univ Paris 06, CNRS,

Département de Chimie, PASTEUR, 24, rue Lhomond, 75005 Paris, France

${ }^{\mathrm{b}}$ Sorbonne Universités, UPMC Univ Paris 06, ENS, CNRS, PASTEUR, 75005 Paris, France

${ }^{\mathrm{c}}$ Univ. Bordeaux, ISM (CNRS UMR 5255), 351 cours de la Libération, 33405 Talence Cedex, France.

\begin{abstract}
The photophysical properties of two highly symmetrical quadrupolar chromophores were studied by both steady-state and transient absorption spectroscopy. Their excited-state behavior is dominated by the solvent-induced Stokes shift of the stimulated-emission band. The origin of this shift is attributed to symmetry breaking that confers a non-vanishing dipole moment to the excited state of both compounds. This dipole moment is large and constant in DMSO, whereas symmetry breaking appears significantly slower and leading to smaller excited-state dipole in toluene. Time-dependant increase of the excited-state dipole moment induced by weak solvation is proposed to explain the results in toluene.
\end{abstract}

\section{Introduction}

Since the 1990s, nondipolar chromophores have been the object of a continued research effort to develop new materials for nonlinear optics. In addition to conventional, seminal conjugated push-pull structures giving rise to nonlinear (second-order) polarizabilities through efficient intramolecular charge transfer (ICT) occurring upon the transition from HOMO to LUMO [1], new families of compounds have emerged that show a higher degree of molecular symmetry. They were aimed at allowing the appearance, and optimization, of nonlinear polarizabilities of more complex origin via non-vanishing elements of the corresponding 
tensors [2]. This has prompted numerous efforts in the design of octupolar derivatives with large optical nonlinear responses, fist aiming at large second-order optical responses (as these derivatives lack a center of symmetry) [3-8] and more recently third-order nonlinear responses [9-12]. In parallel, linear quadrupolar chromophores displaying the generic $D-\pi-A-\pi-D$ or $A-\pi-D-\pi-A$ structure (D standing for electron donor, A for acceptor, and $\pi$ for pi conjugation) attracted particular attention due to their high potentialities for two-photon absorption (2PA) [13-26]. This third-order optical property offers many promises for the development of a variety of technologies (microfabrication, optical limitation, threedimensional data storage, photodynamic therapy, two-photon fluorescence microscopy) [27].

It is worth mentioning here the theoretical framework developed by Terenziani et al. [21] to understand the photophysical and optical properties of quadrupolar chromophores. According to this work, coupling of the zwitterionic valence states of the molecule to nuclear motions localized in each arm of the molecule is capable of inducing symmetry breaking and allows the creation of polar states. Different types of chromophores were defined, exhibiting symmetry breaking either in the one-photon-allowed excited state or in the ground state, depending of the quadrupolar character of the molecule and the strength of the vibronic coupling. Moreover polar solvation was identified as an additional mechanism contributing to the induction of symmetry breaking. This solvent-induced mechanism relies on a classical "slow" (overdamped) solvation coordinate, as opposed to the above-mentioned quantum nuclear coordinates. As a consequence, symmetry cannot be recovered by fast exchange (tunneling) between the two polar minima (false symmetry breaking). Interestingly, several quadrupolar derivatives have been shown to display strong fluorescence solvatochromism despite their symmetrical structure, in relation with symmetry breaking in the excited state [21,28-30].

Ventelon et al. [14,16,17] designed some years ago several series of quadrupolar chromophores for 2PA, which bear a central acceptor group (phenyl or biphenyl or dihydrophenantherene or dithienothiophene) connected on each side to diverse electron donor or acceptor moieties through a semi-rigid conjugated linker (phenylene-vinylene). Some of them were shown to display very large $2 \mathrm{PA}$ cross sections in the visible red or NIR regions. Figure 1 shows two examples of these chromophores, which the present article will deal with. Compound $\mathbf{1}$ has a dihydrophenantherene core while $\mathbf{2}$ has a biphenyl one; both compounds share the same donor, $\mathrm{NBu}_{2}$. 
We report here a study of the photophysical properties of compounds $\mathbf{1}$ and $\mathbf{2}$ by steadystate and picosecond broad-band transient absorption spectroscopy. Our first aim was to characterize the general excited-state behavior of these molecules (lifetime, decay mechanism) and determine for reference purpose their excited-state absorption cross sections over a wide spectral range $(360-800 \mathrm{~nm})$-proposed as Supplementary Information. More specifically, we wished to examine the question of the establishment of symmetry breaking, its dynamics and dependence on the polarity of the solvent. Such questions, discussed on a broad perspective by Vauthey [31], have been tackled recently by ultrafast spectroscopy, for several quadrupolar systems [29,30,32-36]. For the particular D- $\pi$-A- $\pi$-D molecule reported by Dereka et al. [36], the polarity of the solvent was shown to control the nature of the excited state. From symmetrical and quadrupolar in nonpolar solvents, it shifts to a purely dipolar state in highly polar solvents, with full localization of the excitation on one arm of the molecule. An intermediate case is identified in weakly polar media. The dynamics of excitation localization is reported to be controlled by the different time scales of solvent relaxation. In the present study, we used time-dependent Stokes shift of stimulated emission as a reporter of solvation dynamics, itself revealing the effective dipole moment of the molecules in the excited state.

\section{Materials and methods}

\subsection{Samples}

Compound 1 was synthesized as described in Ref. [14] and compound $\mathbf{2}$ as reported in Ref. [37]. The chemical purity of the sample was checked by NMR spectroscopy. Both compounds were found to be in an all-trans configuration, as represented in Figure 1. Spectroscopy grade dimethyl sulfoxide (DMSO) and toluene were purchased from Aldrich and used as received.

\subsection{Transient absorption setup}

Transient absorption experiments were performed by the pump-probe with continuum technique. The source was a non-mode-locked dye laser described elsewhere [38], delivering 500 -fs pulses at $610 \mathrm{~nm}$, with $10 \mathrm{~Hz}$ repetition rate. It was used, through continuum generation, filtering and amplification in dye cells, to produce two synchronous subpicosecond beams, tuned at 570 and $428 \mathrm{~nm}$. 
The pump-probe setup used for the present experiments has been previously described [39]. Excitation of the sample was done with the 428-nm beam while the $570-\mathrm{nm}$ beam generated a continuum of white light used as the probe. The probe beam was delayed with respect to the pump beam in a motorized optical delay line and split into a sample and a reference channel. Pump and probe beams crossed on the sample, held in a 1-mm cuvette, at an angle of ca. $10^{\circ}$ and their linear polarizations were set at the magic angle. The sample solution $(50 \mathrm{~mL})$ was recirculated, at room temperature, to avoid significant photolysis during the experiment. The transmitted beams were sent through optical fibers to the entrance slit a Jobin-Yvon 270M spectrograph (100 grooves/mm grating). Spectra were recorded on a $1024 \times 128$ Princeton Instruments CCD camera and accumulated over 500 pump shots.

Time-resolved differential absorbance spectra $(\Delta \mathrm{A}=$ absorbance of the pumped sample minus absorbance of the unpumped sample) were calculated and corrected for the chirp of the probe pulse (dispersion of the arrival time on the sample), measured in a separate experiment by two-photon absorption in pure 1-chloronaphtalene.

\subsection{Data analysis}

The full transient absorption data, depending on both wavelengths and delay times, were globally fitted [40] to a discrete sum of exponentials, convoluted by a Gaussian representing the instrument response function (IRF). Singular value decomposition (SVD) allowed both dimensional reduction and noise filtering. The rms amplitude of the residue after fitting was of the order of $3 \mathrm{mOD}$. The full width at half maximum of the IRF (time resolution) was found to lie between 1.3 and 1.5 ps.

For each time component of the fit, the collection of wavelength-dependent preexponential factors constitutes a spectrum called decay-associated differential spectrum (DADS).

In order to follow the position of the stimulated-emission band as a function of time, deconvoluted transient absorption spectra, reconstructed from the preceding global multiexponential analysis, were used. The stimulated emission band was fitted by a sum of two lognormal functions, which allowed extracting the wavenumber of its minimum at each time. This position was then plotted as a function of time and fitted to a sum of exponentials.

\section{Results and discussion}




\subsection{Steady-state absorption and fluorescence spectra}

The steady-state absorption and fluorescence spectra of compounds $\mathbf{1}$ and $\mathbf{2}$, in DMSO and toluene, are given in the lower panels of Figures 2 and 3, respectively. Let us recall that DMSO is a strongly polar solvent (dielectric constant $\varepsilon=46.45$, permanent dipole moment $\mu=4.1 \mathrm{D}$ [41]) whereas toluene is nearly nonpolar $(\varepsilon=2.38, \mu=0.3 \mathrm{D}$ [41]). Table 1 gathers the values of the absorption and fluorescence maxima, together with the corresponding fluorescence quantum yields (measured using fluoresceine in $0.1 \mathrm{M} \mathrm{NaOH}$ aqueous solution as a standard). It may be noted that the shape of the fluorescence emission spectra was checked to be independent of the excitation wavelength, for both compounds.

One notes that the solvatochromic shift of the absorption band (shift upon increasing solvent polarity) of both $\mathbf{1}$ and $\mathbf{2}$ is weak ( $\sim 8$ and $14 \mathrm{~nm}$, respectively) and positive (towards longer wavelengths). Conversely, the solvatochromic shift of the fluorescence spectra is very large (80 $\mathrm{nm}$ for $\mathbf{1}$ and $90 \mathrm{~nm}$ for $\mathbf{2}$ ) and positive. This situation evokes the classical case of a molecule bearing a very small permanent dipole moment in the ground state and a large dipole moment in the excited state.

The weakly polar ground state is in fact intuitively expected because of the mostly centrosymmetrical structure of $\mathbf{1}$ and $\mathbf{2}$. It may by the way be noted that the absorption shift of $\mathbf{2}$ is somewhat larger than that of $\mathbf{1}$. One could hypothesize that this is related to the less rigid biphenyl core of $\mathbf{2}$, as compared to the dihydrophenanthrene of $\mathbf{1}$, which possibly allows a slightly larger dissymmetry of the molecule and a larger ground-state dipole moment.

The main issue is however to understand the origin of the polar excited state, given again the symmetrical structure of the molecules. We will for that matter refer to the theoretical framework developed by Terenziani et al. [21] for quadrupolar chromophores (see Introduction). The presently observed solvatochromic behavior corresponds well to class I chromophores, characterized by a low quadrupolar moment in the ground state. For these molecules, the ground state is non polar and displays a single minimum. Symmetry breaking however creates two equivalent polar minima on the one-photon allowed excited-state surface, each of which corresponds to a polar state. For type I chromophores, a further stabilization of these polar broken-symmetry minima by solvation is expected, even in weakly polar solvents, leading to an already important fluorescence solvatochromism in those environments. It may finally be mentioned that, in Terenziani et al.'s model, a lack of mirrorimage symmetry between absorption and fluorescence spectra is expected for class I 
chromophores, even in weakly polar solvents, because of the existence of the double-welled excited state. This is essentially what is observed for $\mathbf{1}$ and $\mathbf{2}$ in toluene (Figures 2C and 3C).

A more detailed study of the solvatochromic behavior of our compounds, in a wider range of solvents, is provided in the Supplementary Information (SI, §1, Figure S1). Figure S2 in particular shows a Lippert-Mataga plot of the steady-state Stokes shift as a function the orientational polarizability of the solvent. Most interestingly, this plot is bimodal: a small slope is found for weakly polar solvents (including toluene), whereas a much larger slope is found for medium to highly polar solvents (including DMSO). This behavior is typical of type I quadrupolar chromophores $[21,25,28]$ and suggests that the effective dipole moment of the excited state is small in toluene and large in DMSO.

\subsection{Transient absorption spectra}

The transient absorption spectra $(\triangle \mathrm{A})$ of compound $\mathbf{1}$ in DMSO are shown in Figures $2 \mathrm{~A}$ and $2 \mathrm{~B}$. For reference, the normalized steady-state absorption and fluorescence spectra, in the same solvent, are given in Figure 2C. Immediately after excitation (1 ps pump-probe delay), negative bands dominated by the ground-state bleaching (GSB) and stimulated emission (SE) contributions are observed, with maxima at around 425 and $512 \mathrm{~nm}$. In addition, a broad, positive, excited-state absorption (ESA) band is seen above $600 \mathrm{~nm}$.

The main spectral evolution observed during the first 20 ps (Figure 2A) is a conspicuous red shift of the SE band (from 512 to $560 \mathrm{~nm}$ ). Meanwhile, a moderate decay of all the bands is observed. At longer delays (Figure 2B) all bands are seen to decay towards zero. It will be shown (section 3.3) that this decay is in fact slightly non-homothetic.

The transient absorption spectra of $\mathbf{1}$ in toluene are displayed in Figures 2D and 2E (steady-state absorption and fluorescence spectra in Figure 2F). Here the GSB band is observed at $410 \mathrm{~nm}$ while the SE band exhibits a well-resolved vibrational structure with major peaks at 445 and $484 \mathrm{~nm}$ (at 1 ps; Figure 2D), consistent with those of the fluorescence spectrum. A broad transient absorption band is seen above $550 \mathrm{~nm}$.

Like in the case of $\mathbf{1}$ in DMSO, a clear Stokes shift of the SE band is observed at short times (below $40 \mathrm{ps}$ ). The amplitude of this shift is however significantly smaller than in the case of DMSO. After $40 \mathrm{ps}$ all bands decay towards zero in a slightly non-homothetic manner. 
The time-resolved differential absorption spectra of compound $\mathbf{2}$ are very similar behavior to those of $\mathbf{1}$, both in DMSO and toluene solutions, and exhibit the same type of temporal behavior. They are shown in Figure 3.

\subsection{Kinetic analysis of the transient absorption data}

We next performed a global multiexponential analysis (see section 2.3 for details) of the transient absorption data. Although this procedure could be applied successfully, it should be kept in mind that it is not ideally suited to describe continuous spectral evolutions, like the time-dependent Stokes shift of stimulated emission usually observed during solvation dynamics [42-45]. Global analysis indeed reduces the data to a superposition of timeindependent spectra, to each of which is attached a given multiexponential kinetics. It is however very useful to quantitatively summarize the overall evolution of the data.

In all cases the data were best fitted with a sum of four exponentials, the time constants of which are summarized in Table 2. The corresponding DADS are given in Figure S3 of the SI $(\S 2)$.

The first two time constants lie in the few ps timescale and it appears clearly that they should be assigned to the Stokes shift dynamics of the stimulated emission, mentioned in section 3.2, which is particularly large in DMSO. A more precise description of this continuous evolution will be given in section 3.4 , with a discussion of its meaning.

The last two time constants are in the hundred of ps to ns regime and correspond to a global decay of the excited state, accompanied by changes of shape of the spectrum. These changes of shape are illustrated in Figure S3, which shows that DADS3 and DADS4 are not simply proportional. An explanation for this non-homothetic biexponential behavior could be sought in the formation of a long-lived photoproduct, such as a triplet state or of a cis-trans isomer, as was for instance shown to be the case for different push-pull polyenes $[46,47]$. We tried to replace the longest-lived exponential by a plateau, standing for the putative photoproduct, but the fits (not shown) were found to be significantly worse and the DADS associated to the plateau did not exhibit convincing features for triplet states or cis-trans isomers. This interpretation was thus not retained. Alternatively, the two time constants could be assigned to the decay of two different and independent species -possibly close conformers already present in the sample solution but not distinguished in the steady-state absorption spectrum. Within this view, DADS3 and DADS4 should be assigned to the excited-state 
decay of each of those species. This interpretation is not convincing as DADS3 and DADS4 show large differences, particularly above $600 \mathrm{~nm}$ in (see Figure S3A). In the case of $\mathbf{1}$ in DMSO, DADS3 does not even display a clear GSB band nor any SE in the region of the fluorescence spectrum. As these differences do not seem justified for closely related species, the hypothesis of heterogeneous decay was ruled out. We finally propose that the biexponential decay observed at long times corresponds to some kind of relaxation of the excited state of the molecules, possibly of geometrical nature. Figure S3 clearly shows that this evolution increases the relative intensity of the ESA band, below $650 \mathrm{~nm}$, as compared to the GSB or SE bands. The exact nature of this phenomenon remains elusive to us.

As a complement for reference purpose, we provide in SI (\$3) spectra of pure excited-state absorption cross sections, corrected from GSB, at an average pump-probe delay of 100 ps (Figure S4).

\subsection{Time-resolved Stokes shift of the stimulated emission}

To interpret the continuous red shift of the SE bands of compounds $\mathbf{1}$ and $\mathbf{2}$ at short pumpprobe delays, it should first be noted that, in all four cases (1 and 2, in DMSO and toluene), the excitation wavelength was chosen to lie (slightly or markedly) on the red side of the lowest absorption band. The vibrational energy excess upon excitation is thus guaranteed to be null or small. Vibrational cooling was therefore not considered as a suitable explanation. Solvation dynamics, i.e. the reorganization of the solvation cage as the electronic distribution of the molecule is modified by excitation, is a much more likely option. It is indeed very well known to give rise to time-resolved Stokes shifts of the fluorescence or of the stimulated emission $[42-45,48,49]$.

In order to investigate this interpretation further, we determined the frequency (in wavenumbers) of the SE minimum as a function of pump-probe delay (see section 2.3 for details). These data were then fitted by a sum of exponential functions. Figure 4 shows the corresponding plots and Table 3 gathers the time constants and associated pre-exponential factors.

Let us first compare the time constants of our fits of the SE shifts with the reported time constants of solvation dynamics, measured by time-resolved fluorescence spectroscopy of coumarin 153 (C153), a standard probe of polar solvation dynamics [48,49]. Since the 
ultrashort time components reported by the Maroncelli group $(<1 \mathrm{ps})$ were not accessible to our setup, we compare here the longest ones, lying in the ps regime.

For DMSO, the time constants of 1 (2.0 and $12 \mathrm{ps)}$ are in good agreement with those of C153, namely 2.3 and 10.7 ps [48]. The weighted average time constant, taking into account the amplitude of each component, is $3.0 \mathrm{ps}$ for $\mathbf{1}$, which is also comparable to the value of 3.8 ps calculated by weight-averaging the ps time constants of $\mathrm{C} 153$. The same global agreement is found for 2 , the individual time constants of which being 1.5 and $12.5 \mathrm{ps}$, and the averaged value $2.9 \mathrm{ps}$. In the case of toluene, the characteristic times of $\mathbf{1}$ (1.1 and $8.8 \mathrm{ps}$ ) are significantly shorter than the homologous ones reported for C153, 2.2 and 15.2 ps [49]. The mismatch is however less pronounced upon comparing the weight-averaged values, $3.8 \mathrm{ps}$ for 1 and 4.3 ps for C153. This is due to the fact that slowest component of the shift dynamics has more weight in $\mathbf{1}$ than in $\mathrm{C} 153$. For $\mathbf{2}$, only one time constant (4.8 ps) was found, rather close to the weighted average of $\mathrm{C} 153$. The fact that we could not resolve a shorter time constant again suggests that the shift dynamics of $\mathbf{2}$ is more centered on slow components than that for C153.

Turning now to the total amplitude of the SE shift, we may observe that it is rather large in DMSO, $2539 \mathrm{~cm}^{-1}$ for 1 and $2630 \mathrm{~cm}^{-1}$ for 2 . The homologous shift amplitude for $\mathrm{C} 153$, calculated with the ps components only, is $1060 \mathrm{~cm}^{-1}$ [48]. Taking C153 as a reference of dipolar solvation, let us note that the ratio of shift amplitudes to $\mathrm{C} 153(R)$ is 2.39 and 2.48 , for 1 and 2 respectively. Regarding toluene, we observe a much smaller total shift, of $370 \mathrm{~cm}^{-1}$ for 1 and $222 \mathrm{~cm}^{-1}$ for 2 . In this solvent, the reference value of $\mathrm{C} 153$ is $571 \mathrm{~cm}^{-1}$, calculated again with the ps components only [49]. The $R$ ratio becomes here 0.65 and 0.39 , for 1 and 2 respectively. It appears thus quite clearly that $\mathbf{1}$ and $\mathbf{2}$ behave very differently in DMSO and toluene.

From the above comparisons to $\mathrm{C} 153$, we confirm on the one hand that the time-dependent shift which we observed for the SE band is due to solvation dynamics. A very good agreement is indeed found at all levels (time constants and relative amplitudes) in DMSO. This further indicates that $\mathbf{1}$ and $\mathbf{2}$ behave in this solvent just as any ordinary molecule instantly gaining dipole moment upon electronic excitation. Within the theoretical framework of Terenziani et al. [21], this means that the full extent of the symmetry-broken, excited-state dipole moment is extremely quickly reached in DMSO, most likely in during the sub-ps fraction of the solvation response (214 fs in DMSO [48]). The solvation dynamics observed in the ps regime is therefore indistinguishable from that of a standard polar probe. These conclusions agree 
qualitatively well with the observations of Dereka et al. [36] (see Introduction) for a comparable quadrupolar probe in highly polar solvents. They indeed report the conversion from a quadrupolar state to an intermediately polar state in $0.8 \mathrm{ps}$ in DMSO, followed by further evolution to a dipolar state in $2.5 \mathrm{ps}$. In the particular case of $\mathbf{1}$ and $\mathbf{2}$, it may be hypothesized that the fully (or at least steady) dipolar state would be reached faster.

A more complex and intriguing behavior is observed in toluene. The fact that the shift dynamics of 1 and $\mathbf{2}$ contain lesser amounts of short-time component first suggests that the dipole moment of the excited state has not reached its full amplitude at the shortest observation times of our study. One may suppose that it increases with time, giving thereby more weight to the slowest components of the solvation response. On the other hand, the fact that the total amplitude of the shift becomes smaller than that of $\mathrm{C} 153$ in toluene $(R<0.7)$, while it is more than twice bigger in DMSO $(R \geq 2.4)$, most probably indicates that the final magnitude of the excited-state dipole moment is significantly smaller in toluene than in DMSO.

Again, our conclusions agree rather well with those of Dereka et al. [36] for their quadrupolar compound in weakly polar media. They indeed similarly report the formation of an intermediate state with partial localization of the excitation on one arm of the molecule, and consequently smaller dipole moment than in highly polar solvents.

The above difference between toluene and DMSO in fact fully agrees with our observation of a sharp discontinuity in the Lippert-Mataga plot of the steady-state Stokes shift of our compounds (SI, §1, Figure S2). It is interesting to note that the ratio of the Lippert-Mataga slopes at high and low polarities (of the order of 7.5) is commensurable with the ratio of $R$ values between DMSO and toluene (3.7 for 1 and 6.4 for 2), indicating that both observations (steady-state and time-resolved Stokes shift) relate to the same phenomenon.

Globally our findings may be qualitatively understood within the framework of Terenziani et al. [21], which views dipolar solvation as cooperating with vibrational coupling to induce symmetry breaking. It is thus not surprising that the more polar DMSO supports a larger dipolar distortion of the molecule than toluene. One can further propose that the depth of the polar excited-state minima is never large enough in toluene, even at completion of the solvation process, to preclude some partial recovery of inversion symmetry through tunneling along the anti-symmetric nuclear coordinate (see section 3.1). Toluene would thus exemplify a case of false (or nearly false) symmetry breaking. Conversely, DMSO would be polar enough to impair this putative exchange mechanism, even shortly after the onset of the 
solvation process, and to allow the appearance of a full symmetry breaking, with charge separation occurring in one arm only of the molecule.

\section{Conclusion}

Steady-state absorption and fluorescence spectroscopy were used to characterize the basic photophysical properties of compounds $\mathbf{1}$ and $\mathbf{2}$. The small absorption solvatochromism, and correlatively large fluorescence solvatochromism of the compounds indicate that the ground state is weakly polar and the relaxed excited state is highly polar. Given that $\mathbf{1}$ and $\mathbf{2}$ are highly symmetrical molecules, this seemingly trivial situation in fact reveals that a nonstandard process of symmetry breaking takes place in the excited state. Within the theoretical framework developed by Terenziani et al. [21] for quadrupolar chromophores, we propose that both 1 and $\mathbf{2}$ should be classified into type I chromophores, bearing small quadrupolar moment.

Using picosecond transient absorption spectroscopy, we have shown that the excited-state behavior of $\mathbf{1}$ and $\mathbf{2}$ is dominated by the solvent-induced Stokes shift of the stimulatedemission band, occurring in the few-ps time scale. Longer-lived components of the evolution were detected in the few-hundred-ps regime and tentatively assigned to some yet undefined structural relaxation of the molecules.

A dedicated study of the time-dependent Stokes shift of the stimulated-emission band demonstrated that the solvation dynamics of $\mathbf{1}$ and $\mathbf{2}$ in DMSO is essentially identical, in the ps time scale, to the one of coumarin 153 in the same solvent. Significant differences are however found in toluene, where the fast solvation components of $\mathbf{1}$ and $\mathbf{2}$ appear attenuated. Significantly, the total amplitude of the shift is found to be smaller than that of coumarin 153 in toluene while it is much larger in DMSO. These observations are understood in terms of magnitude of the excited-state dipole moment. The full extent of it appears to be reached in the sub-ps regime in DMSO while a progressive increase of its value, reaching a smaller maximum, is supposed to be at work in toluene. This interpretation is justified within the Terenziani et al. model by the cooperation of both vibronic coupling and polar solvation to induce symmetry breaking and stabilize the excited-state polar minima. The polarity of the solvent is thus expected to play a crucial role in determining the dipole moment of the excited state. It may additionally be hypothesized that partial recovery of inversion symmetry through 
tunneling could also contribute to the relatively small effective excited-state dipole moment in toluene.

Supporting Information Available. Contents: 1) Solvatochromism; 2) DADS of the global multiexponential analysis; 3) Transient absorption cross sections.

\section{References}

[1] J.L. Oudar, D.S. Chemla, J. Chem. Phys. 66 (1977) 2664.

[2] J. Zyss, I. Ledoux, Chem. Rev. 94 (1994) 77.

[3] J.J. Wolff, D. Langle, D. Hillenbrand, R. Wortmann, R. Matschiner, C. Glania, P. Kramer, Adv. Mat. 9 (1997) 138.

[4] R. Wortmann, C. Glania, P. Kramer, R. Matschiner, J.J. Wolff, S. Kraft, B. Treptow, E. Barbu, D. Langle, G. Gorlitz, Chem. Eur. J. 3 (1997) 1765.

[5] C. Lambert, E. Schmalzlin, K. Meerholz, C. Brauchle, Chem. Eur. J. 4 (1998) 512.

[6] S. Brasselet, F. Cherioux, P. Audebert, J. Zyss, Chem. Mat. 11 (1999) 1915.

[7] J.J. Wolff, F. Siegler, R. Matschiner, R. Wortmann, Angew. Chem.Int. Ed. 39 (2000) 1436.

[8] J. Brunel, O. Mongin, A. Jutand, I. Ledoux, J. Zyss, M. Blanchard-Desce, Chem. Mat. 15 (2003) 4139.

[9] D. Beljonne, W. Wenseleers, E. Zojer, Z.G. Shuai, H. Vogel, S.J.K. Pond, J.W. Perry, S.R. Marder, J.L. Bredas, Adv. Funct. Mat. 12 (2002) 631.

[10] C. Katan, F. Terenziani, O. Mongin, M.H.V. Werts, L. Porres, T. Pons, J. Mertz, S. Tretiak, M. Blanchard-Desce, J. Phys. Chem. A 109 (2005) 3024.

[11] Y.M. Poronik, V. Hugues, M. Blanchard-Desce, D.T. Gryko, Chem. Eur. J. 18 (2012) 9258.

[12] G. Argouarch, R. Veillard, T. Roisnel, A. Amar, H. Meghezzi, A. Boucekkine, V. Hugues, O. Mongin, M. Blanchard-Desce, F. Paul, Chem. Eur. J. 18 (2012) 11811. 
[13] M. Albota, D. Beljonne, J.L. Bredas, J.E. Ehrlich, J.Y. Fu, A.A. Heikal, S.E. Hess, T. Kogej, M.D. Levin, S.R. Marder, D. McCord-Maughon, J.W. Perry, H. Rockel, M. Rumi, C. Subramaniam, W.W. Webb, X.L. Wu, C. Xu, Science 281 (1998) 1653.

[14] L. Ventelon, L. Moreaux, J. Mertz, M. Blanchard-Desce, Chem. Comm. (1999) 2055.

[15] M. Barzoukas, M. Blanchard-Desce, J. Chem. Phys. 113 (2000) 3951.

[16] L. Ventelon, S. Charier, L. Moreaux, J. Mertz, M. Blanchard-Desce, Angew. Chem.Int. Ed. 40 (2001) 2098.

[17] L. Ventelon, L. Moreaux, J. Mertz, M. Blanchard-Desce, Synth. Metals 127 (2002) 17.

[18] W.J. Yang, D.Y. Kim, M.Y. Jeong, H.M. Kim, S.J. Jeon, B.R. Cho, Chem. Comm. (2003) 2618.

[19] K.D. Belfield, A.R. Morales, B.S. Kang, J.M. Hales, D.J. Hagan, E.W. Van Stryland, V.M. Chapela, J. Percino, Chem. Mat. 16 (2004) 4634.

[20] M. Charlot, L. Porres, C.D. Entwistle, A. Beeby, T.B. Marder, M. Blanchard-Desce, Phys. Chem. Chem. Phys. 7 (2005) 600.

[21] F. Terenziani, A. Painelli, C. Katan, M. Charlot, M. Blanchard-Desce, J. Am. Chem. Soc. 128 (2006) 15742.

[22] O. Mongin, L. Porres, M. Charlot, C. Katan, M. Blanchard-Desce, Chem. Eur. J. 13 (2007) 1481.

[23] F. Terenziani, C. Katan, E. Badaeva, S. Tretiak, M. Blanchard-Desce, Adv. Mat. 20 (2008) 4641.

[24] J.C. Collings, S.-Y. Poon, C. Le Droumaguet, M. Charlot, C. Katan, L.-O. Palsson, A. Beeby, J.A. Mosely, H.M. Kaiser, D. Kaufmann, W.-Y. Wong, M. Blanchard-Desce, T.B. Marder, Chem. Eur. J. 15 (2009) 198.

[25] C. Katan, M. Charlot, O. Mongin, C. Le Droumaguet, V. Jouikov, F. Terenziani, E. Badaeva, S. Tretiak, M. Blanchard-Desce, J. Phys. Chem. B 114 (2010) 3152.

[26] I. Baglai, M. de Anda-Villa, R.M. Barba-Barba, C. Poidevin, G. Ramos-Ortiz, V. Maraval, C. Lepetit, N. Saffon-Merceron, J.-L. Maldonado, R. Chauvin, Chem. Eur. J. 21 (2015) 14186.

[27] M. Pawlicki, H.A. Collins, R.G. Denning, H.L. Anderson, Angew. Chem.Int. Ed. 48 (2009) 3244.

[28] S. Amthor, C. Lambert, S. Dummler, I. Fischer, J. Schelter, J. Phys. Chem. A 110 (2006) 5204. 
[29] P.D. Zoon, I.H.M. van Stokkum, M. Parent, O. Mongin, M. Blanchard-Desce, A.M. Brouwer, Phys. Chem. Chem. Phys. 12 (2010) 2706.

[30] B. Carlotti, E. Benassi, C.G. Fortuna, V. Barone, A. Spalletti, F. Elisei, ChemPhysChem 17 (2016) 136.

[31] E. Vauthey, ChemPhysChem 13 (2012) 2001.

[32] A. Bhaskar, G. Ramakrishna, Z. Lu, R. Twieg, J.M. Hales, D.J. Hagan, E. Van Stryland, T. Goodson, III, J. Am. Chem. Soc. 128 (2006) 11840.

[33] G. Ramakrishna, T. Goodson, III, J. Phys. Chem. A 111 (2007) 993.

[34] S. Easwaramoorthi, P. Thamaraiselvi, K. Duraimurugan, A.J. Beneto, A. Siva, B.U. Nair, Chem. Comm. 50 (2014) 6902.

[35] B. Carlotti, E. Benassi, A. Spalletti, C.G. Fortuna, F. Elisei, V. Barone, Phys. Chem. Chem. Phys. 16 (2014) 13984.

[36] B. Dereka, A. Rosspeintner, Z. Li, R. Liska, E. Vauthey, J. Am. Chem. Soc. 138 (2016) 4643.

[37] L. Ventelon, L. Porres, M. Blanchard-Desce, Y. Morel, P. Baldeck, P. Feneyrou, M. Silly, P.A. Chollet, Proc. SPIE 4797 (2003) 15.

[38] N.D. Hung, P. Plaza, M. Martin, Y.H. Meyer, Appl. Optics 31 (1992) 7046.

[39] Y.H. Meyer, P. Plaza, Chem. Phys. 200 (1995) 235.

[40] I.H.M. van Stokkum, D.S. Larsen, R. van Grondelle, Biochim. Biophys. Acta Bioenerg. 1657 (2004) 82.

[41] J.A. Riddick, W.B. Bunger, T.K. Sakano, Organic Solvents, Wiley-Interscience, 1986.

[42] D. Bingemann, N.P. Ernsting, J. Chem. Phys. 102 (1995) 2691.

[43] J. Ruthmann, S.A. Kovalenko, N.P. Ernsting, D. Ouw, J. Chem. Phys. 109 (1998) 5466.

[44] P. Changenet-Barret, C.T. Choma, E.F. Gooding, W.F. DeGrado, R.M. Hochstrasser, J. Phys. Chem. B 104 (2000) 9322.

[45] C. Ley, J. Brazard, F. Lacombat, P. Plaza, M.M. Martin, G.A. Kraus, J.W. Petrich, Chem. Phys. Lett. 457 (2008) 82.

[46] D. Laage, P. Plaza, M. Blanchard-Desce, M.M. Martin, Photochem. Photobiol. Sci. 1 (2002) 526.

[47] W. Akemann, D. Laage, P. Plaza, M.M. Martin, M. Blanchard-Desce, J. Phys. Chem. B $112(2008) 358$. 
[48] M.L. Horng, J.A. Gardecki, A. Papazyan, M. Maroncelli, J. Phys. Chem. 99 (1995) 17311.

[49] L. Reynolds, J.A. Gardecki, S.J.V. Frankland, M.L. Horng, M. Maroncelli, J. Phys. Chem. 100 (1996) 10337. 


\section{TABLES}

\section{Table 1}

Absorption and fluorescence maxima ( $\lambda_{\text {abs }}$ and $\lambda_{\text {fluo }}$, respectively) of compounds $\mathbf{1}$ and $\mathbf{2}$, in DMSO and toluene. The corresponding fluorescence quantum yields $\left(\Phi_{\text {fluo }}\right)$, measured against fluoresceine in $0.1 \mathrm{M} \mathrm{NaOH}$ aqueous solution, are given in the last column.

\begin{tabular}{cllll}
\hline Compound & Solvent & $\lambda_{\text {abs }}(\mathrm{nm})$ & $\lambda_{\text {fluo }}(\mathrm{nm})$ & $\Phi_{\text {fluo }}$ \\
\hline $\mathbf{1}$ & Toluene & $412.7 \pm 0.2$ & $456.4 \pm 0.2$ & 0.71 \\
& DMSO & $420.5 \pm 0.6$ & $536.5 \pm 0.5$ & 0.87 \\
\hline \multirow{2}{*}{} & Toluene & $399.3 \pm 0.3$ & $455.4 \pm 0.3$ & 0.81 \\
& DMSO & $412.9 \pm 0.3$ & $545.7 \pm 1.0$ & 0.89 \\
\hline
\end{tabular}

\section{Table 2}

Time constants obtained by global multiexponential analysis of the transient absorption data.

\begin{tabular}{clllll}
\hline Compound & Solvent & $\tau_{1}(\mathrm{ps})$ & $\tau_{2}(\mathrm{ps})$ & $\tau_{3}(\mathrm{ps})$ & $\tau_{4}(\mathrm{ps})$ \\
\hline \multirow{2}{*}{} & Toluene & $1.3 \pm 0.2$ & $8.2 \pm 1.3$ & $250 \pm 14$ & $840 \pm 33$ \\
& DMSO & $1.0 \pm 0.2$ & $5.3 \pm 0.6$ & $549 \pm 285$ & $987 \pm 226$ \\
\hline \multirow{2}{*}{} & Toluene & $2.9 \pm 1$ & $15 \pm 3$ & $298 \pm 43$ & $780 \pm 57$ \\
& DMSO & $0.85 \pm 0.1$ & $5.6 \pm 0.5$ & $745 \pm 150$ & $1902 \pm 445$ \\
\hline
\end{tabular}

\section{Table 3}

Time-dependent Stokes shift of the SE band. The frequency of the maximum of the band has been fitted to a sum of exponentials. The time constants $\left(\tau_{\mathrm{i}}\right)$ corresponding pre-exponential factors $\left(\mathrm{a}_{\mathrm{i}}\right)$ and the weighted average time constant $(\langle\tau\rangle)$ are indicated. Standard errors are provided for the time constants only.

\begin{tabular}{lllllll}
\hline Solv. & Comp. & $\tau_{1}(\mathrm{ps})$ & $\mathrm{a}_{1}\left(\mathrm{~cm}^{-1}\right)$ & $\tau_{2}(\mathrm{ps})$ & $\mathrm{a}_{2}\left(\mathrm{~cm}^{-1}\right)$ & $\langle\tau\rangle(\mathrm{ps})$ \\
\hline DMSO & $\mathbf{1}$ & $2.0 \pm 0.2$ & 2296 & $12 \pm 7$ & 243 & 3.0 \\
& $\mathbf{2}$ & $1.5 \pm 0.3$ & 2290 & $12.5 \pm 13.5$ & 341 & 2.9 \\
\hline Toluene & $\mathbf{1}$ & $1.1 \pm 0.1$ & 241 & $8.8 \pm 0.7$ & 130 & 3.8 \\
& $\mathbf{2}$ & $4.8 \pm 0.1$ & 222 & - & - & 4.8 \\
\hline
\end{tabular}




\section{FIGURES}
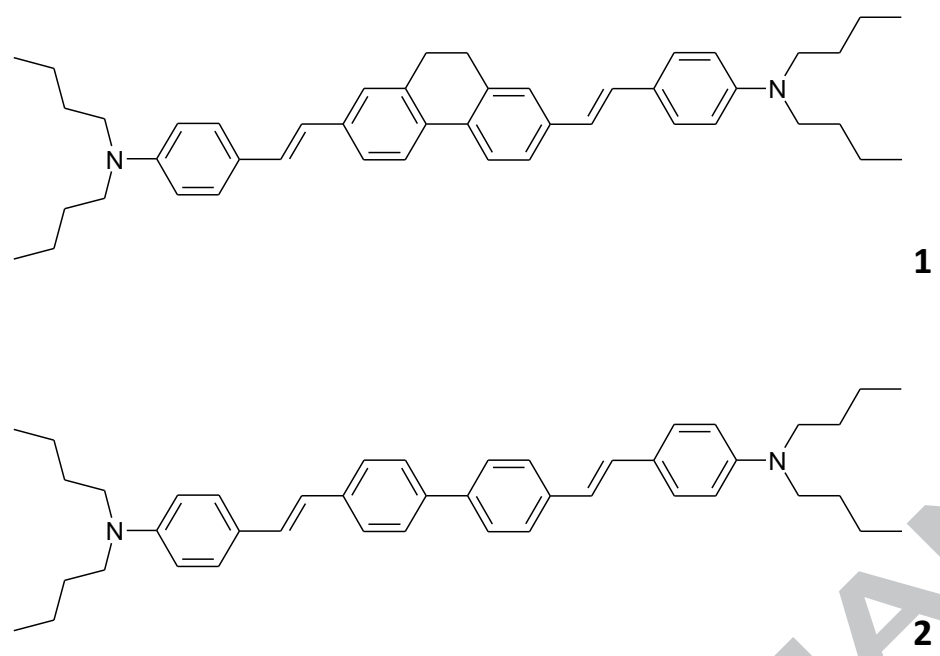

Figure 1. Structures of the push-push quadrupolar chromophores. 
1 in DMSO

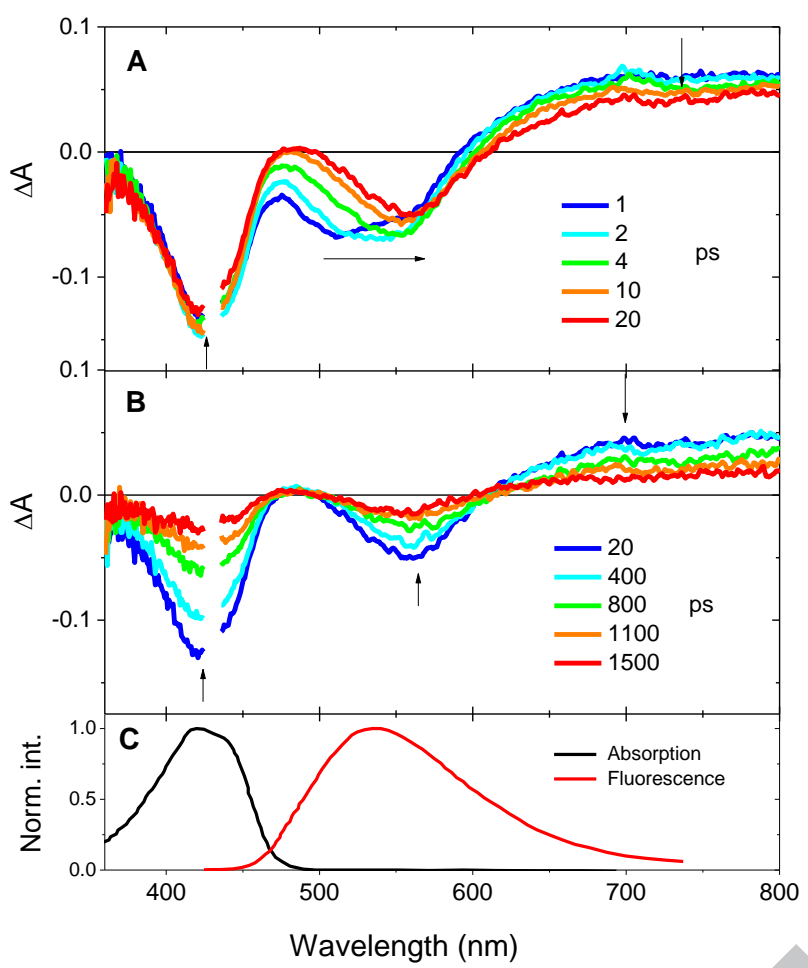

1 in toluene

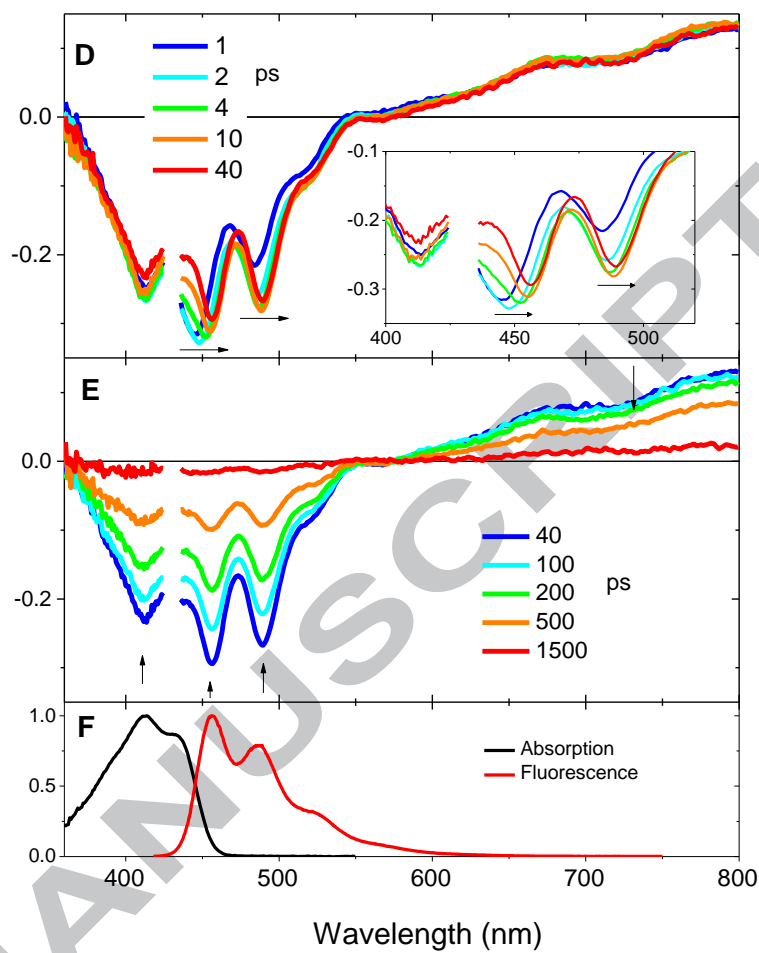

Figure 2. Transient absorption spectra of 1 in DMSO (left column) and in toluene (right column), after sub-ps excitation at $430 \mathrm{~nm}$, at different pump-probe delays. The lower part of the figure gives the steady-state absorption (gray) and fluorescence (red) spectra of the molecule. 
2 in DMSO

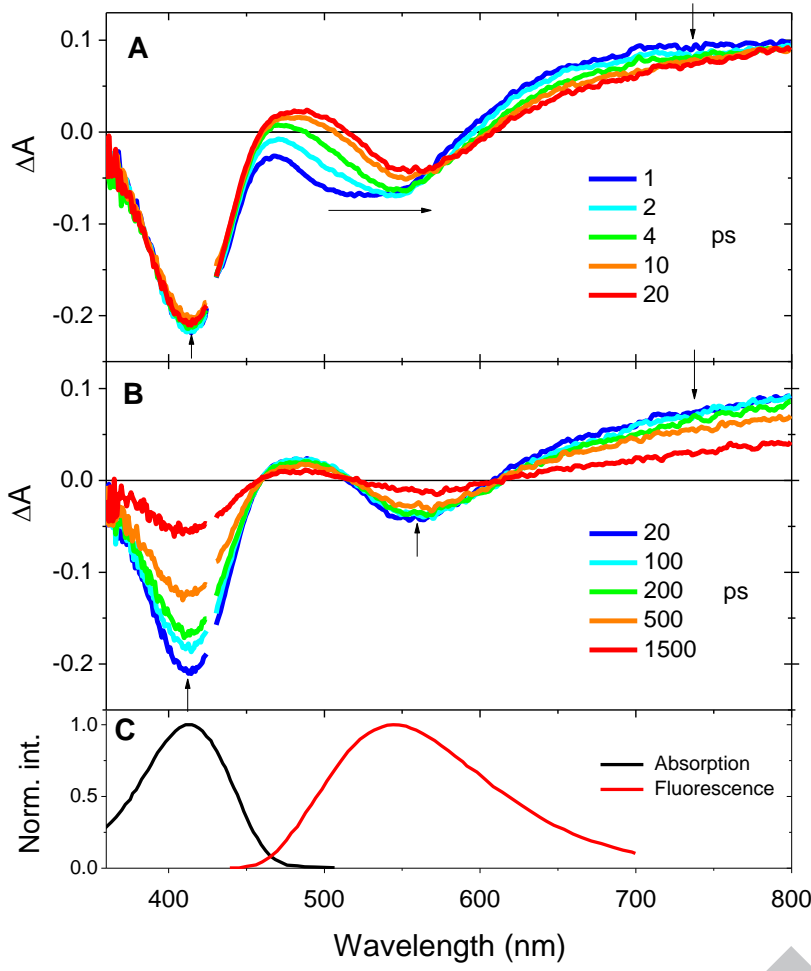

2 in toluene

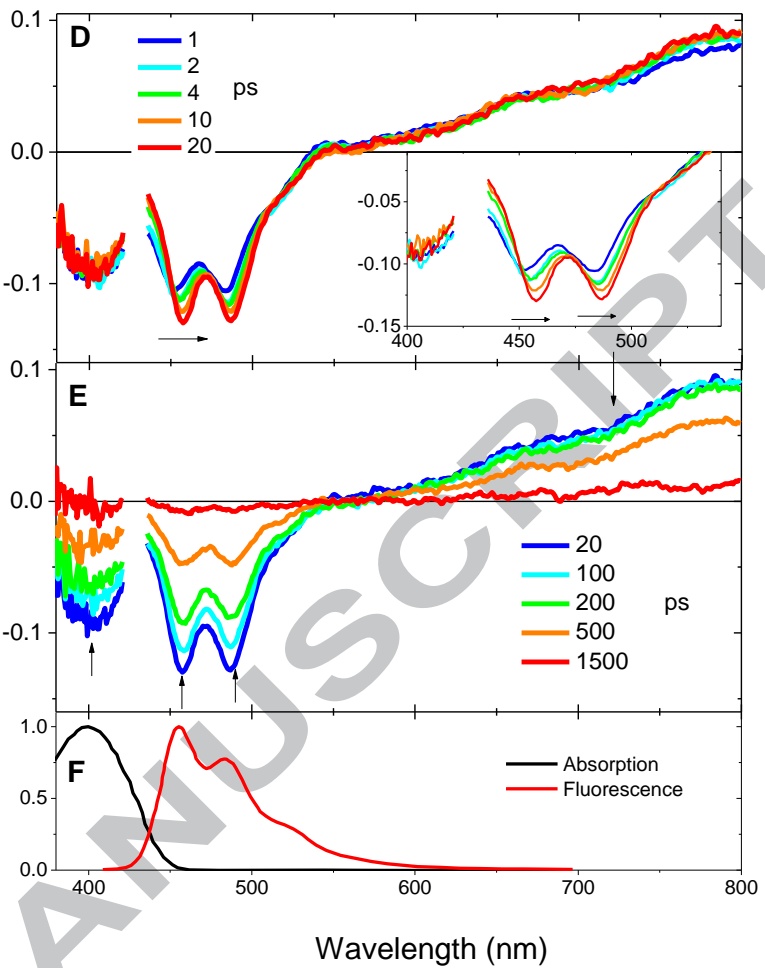

Figure 3. Transient absorption spectra of 2 in DMSO (left column) and in toluene (right column), after sub-ps excitation at $430 \mathrm{~nm}$, at different pump-probe delays. The lower part of the figure gives the steady-state absorption (gray) and fluorescence (red) spectra of the molecule. 

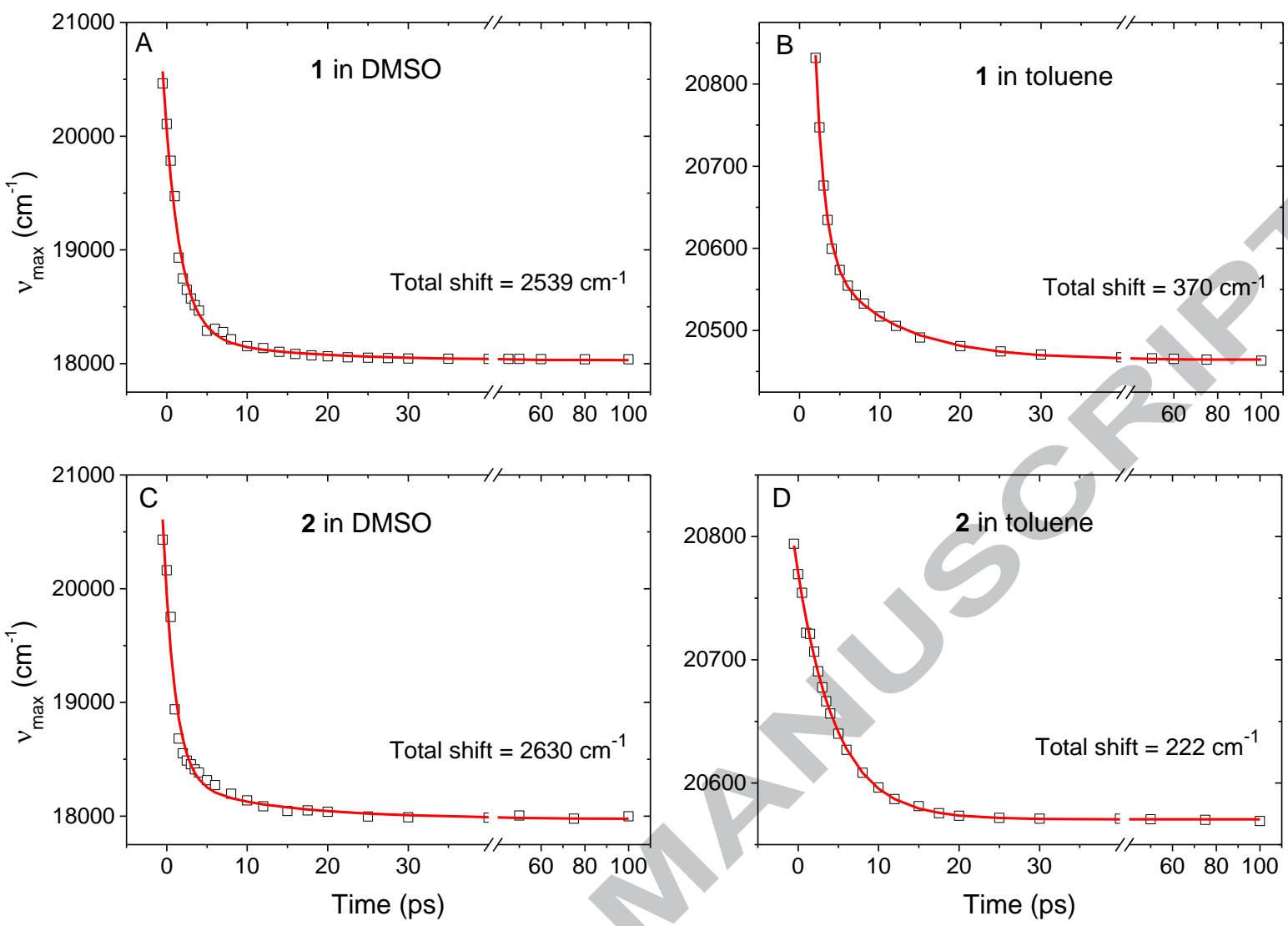

Figure 4. Time-dependent shift of the SE band maximum ( $\left.v_{\max }\right)$ of $\mathbf{1}$ in DMSO (A), 1 in toluene (B), 2 in DMSO (C) and 2 in toluene (D). The data have been fitted to a sum of exponentials (red line), the parameters of which are gathered in Table 3. 


\section{Graphical Abstract}
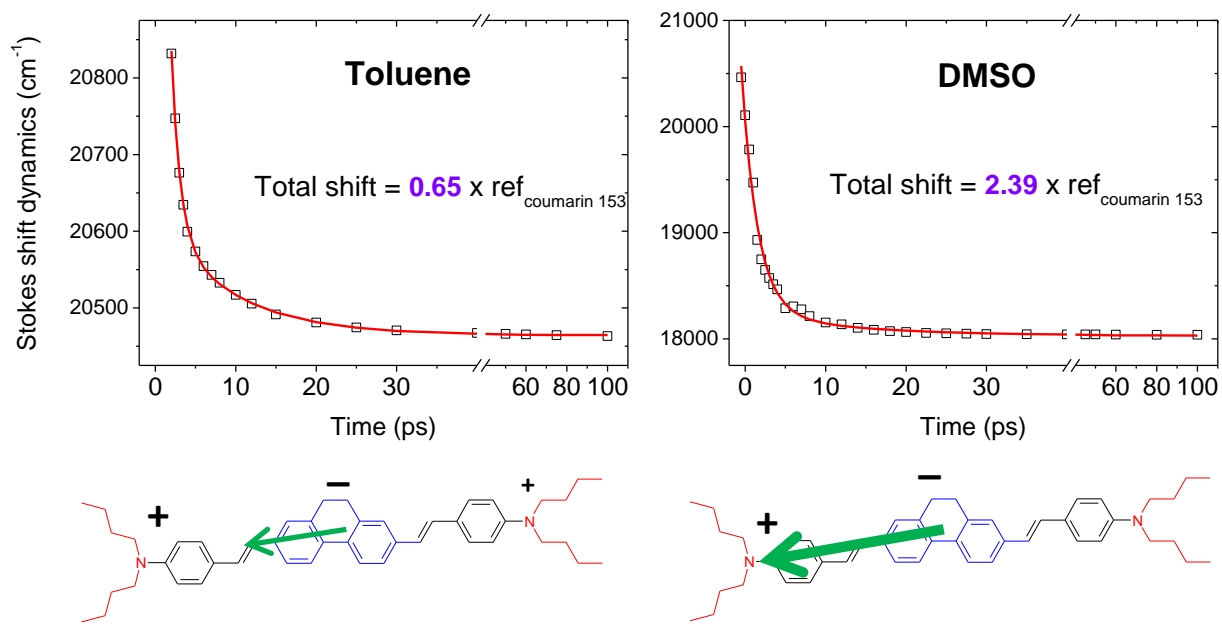

Excited-state dipole moment 


\section{Highlights}

- Transient absorption spectroscopy of symmetrical quadrupolar chromophores

- Stokes shift dynamics of stimulated emission reveals excited state dipole moment

- Symmetry breaking confers non-vanishing dipole moment to excited state

- Large and fast symmetry breaking in DMSO; slower and smaller effect in toluene 